\title{
Functional assessment of coronary artery flow using adenosine stress dual-energy CT: a preliminary study
}

\author{
Michinobu Nagao - Teruhito Kido Kouki Watanabe \\ Hideyuki Saeki · Hideki Okayama • Akira Kurata • \\ Kohei Hosokawa · Hiroshi Higashino · Teruhito Mochizuki
}

Received: 14 March 2010/Accepted: 24 July 2010/Published online: 5 August 2010

(C) The Author(s) 2010. This article is published with open access at Springerlink.com

\begin{abstract}
We attempted to assess coronary artery flow using adenosine-stress and dual-energy mode with dual-source CT (DE-CT). Data of 18 patients with suspected coronary arteries disease who had undergone cardiac DE-CT were retrospectively analyzed. The patients were divided into two groups: 10 patients who performed adenosine stress CT, and 8 patients who performed rest CT as controls. We reconstructed an iodine map and composite images at $120 \mathrm{kV}$ (120 kV images) using raw data with scan parameters of 100 and $140 \mathrm{kV}$. We measured mean attenuation in the coronary artery proximal to the distal portion on both the iodine map and $120 \mathrm{kV}$ images. Coronary enhancement ratio (CER) was calculated by dividing
\end{abstract}

M. Nagao ( $\bowtie)$

Department of Molecular Imaging and Diagnosis, Graduate School of Medical Sciences, Kyushu University, 3-1-1 Maidashi, Higashi-Ku, Fukuoka-City, Fukuoka 812-8582, Japan e-mail: minagao@radiol.med.kyushu-u.ac.jp

T. Kido - A. Kurata - K. Hosokawa $\cdot$ H. Higashino ·

T. Mochizuki

Department of Radiology, Ehime University Graduate

School of Medicine, Toon-City, Japan

K. Watanabe $\cdot$ H. Saeki

Department of Cardiology, Saiseikai Matsuyama

Hospital, Matsuyama-City, Japan

H. Okayama

Department of Cardiology, Ehime University Graduate

School of Medicine, Toon-City, Japan mean attenuation in the coronary artery by attenuation in the aortic root, and was used as an estimate of coronary enhancement. Coronary stenosis was identified as a reduction in diameter of $>50 \%$ on CT angiogram, and myocardial ischemia was diagnosed by adenosine-stress myocardial perfusion scintigraphy. The iodine map showed that CER was significantly lower for ischemic territories $(0.76 \pm 0.06)$ or stenosed coronary arteries $(0.77 \pm 0.06)$ than for nonischemic territories $(0.95 \pm 0.21, P=0.02)$ or nonstenosed coronary arteries $(1.07 \pm 0.33, P<0.001)$. The $120 \mathrm{kV}$ images showed no difference in CER between these two groups. Use of CER on the iodine map separated ischemic territories from non-ischemic territories with a sensitivity of $86 \%$ and a specificity of $75 \%$. Our quantification is the first non-invasive analytical technique for assessment of coronary artery flow using cardiac CT. CER on the iodine map is a candidate method for demonstration of alteration in coronary artery flow under adenosine stress, which is related to the physiological significance of coronary artery disease.

Keywords Dual-energy CT · CT coronary angiogram $\cdot$ Adenosine $\cdot$ Coronary artery flow

\section{Introduction}

Currently, 64-slice cardiac computed tomography (CT) scanners have the ability to completely assess 
the entire coronary tree and have been demonstrated to have good diagnostic accuracy in the identification of anatomically important coronary artery disease (CAD), which is generally defined as coronary artery stenosis with a lumen diameter reduction of at least $50 \%[1,2]$. However, anatomically significant coronary stenosis is not always indicative of functional stenosis: this is particularly true for intermediate-type coronary lesions [3-5]. According to established guidelines (European Society of Cardiology, American College of Cardiology/American Heart Association), the decision to perform angioplasty or bypass surgery should integrate anatomical information with a test that provides objective proof of ischemia $[6,7]$.

Although the recently introduced dual-source CT scanner was developed primarily to achieve high temporal resolution in cardiac imaging [8, 9], it has resolved a primary drawback of contrast-enhanced applications as well, since it enables dual-energy differentiation [10]. Contrast-enhanced dual-energy mode with dual-source CT (DE-CT) can depict abnormal pulmonary blood distribution in pulmonary embolism [11]. Furthermore, adenosine stress cardiac DE-CT can detect myocardial ischemia using the myocardial blood pool images $[12,13]$. We therefore hypothesized that cardiac DE-CT can demonstrate alterations in coronary artery flow that are induced by adenosine stress. The aim of the present study was to assess coronary artery flow using adenosine stress DE-CT.

\section{Materials and methods}

Our Human Research Committee approved this retrospective analysis and waived informed patient consent. This study was conducted in compliance with the Health Insurance Portability and Accountability Act.

\section{Patients}

Data for 18 consecutive patients who had undergone cardiac DE-CT were retrospectively analyzed. The patients were divided into two groups: 10 patients (7 men, 3 women; age range, 61-77 years; mean age, 70 years) who underwent adenosine stress CT, and 8 patients ( 4 men, 4 women; age range, 55-82 years; mean age, 72 years) who underwent rest $\mathrm{CT}$ as controls. All patients had been clinically referred for assessment of known or suspected CAD. The entry criteria were as follows: (1) effort or rest stable angina (documented ST-T change on electrocardiogram (ECG), or relief with administration of nitroglycerin); and (2) asymptomatic patients with a high probability of coronary artery disease (i.e., multiple coronary risk factors) or abnormal findings on exercise ECG. The exclusion criteria were as follows: (1) acute myocardial infarction (within 3 months); (2) unstable angina (recent onset of angina within 1 month, severe and worsening clinical symptoms); (3) chronic atrial fibrillation; (4) poor renal function (serum creatinine $>1.5 \mathrm{mg} / \mathrm{dl}$ ); (5) pregnancy, hyperthyroidism, or known allergic reaction to contrast media; (6) severe left ventricular dysfunction (left ventricular ejection fraction less than 20\%); (7) known history of bronchial asthma; (8) congestive heart failure (New York Heart Association class IV); and (9) greater than first-degree atrio-ventricular block.

Coronary risk factors among the patients were as follows: hypertension (14 patients), diabetes mellitus (6), dyslipidemia (7), and cigarette smoking (6).

To decide whether to perform angioplasty or bypass surgery following DE-CT studies, adenosine stress myocardial perfusion scintigraphy (MPS) was performed in 10 (7 patients in adenosine stress CT group, 3 in rest CT group) of the 18 patients. Invasive coronary angiography (ICA) was performed in 8 patients ( 5 in adenosine stress CT group, 3 in rest CT group).

\section{CT procedures}

All patients were examined using a dual-source CT system (Definition, Siemens, Forchheim, Germany) in dual-energy mode. In each patient, a single CT acquisition was obtained with the following parameters: $330 \mathrm{~ms}$ gantry rotation time, $32 \times 2 \times 0.6-\mathrm{mm}$ collimation with z-flying focal spot technique [14] and $165 \mathrm{~ms}$ temporal resolution. Helical pitch was 0.2 (heart rate: $<60$ beats/min), 0.25 (61-70 beats/min), 0.3 ( $71-80$ beats $/ \mathrm{min})$, and 0.35 ( $>81$ beats/min). One tube of the dual-source CT system was operated at $90 \mathrm{mAs} / \mathrm{rot}$ at $140 \mathrm{kV}$ and the second tube at $180 \mathrm{mAs} / \mathrm{rot}$ at $100 \mathrm{kV}$. Data were acquired in the cranio-caudal direction with simultaneous recording of the patient's ECG signal to enable retrospective 
registration of the reconstructed images to the desired cardiac phase. The anatomical range extended from the level of the carina to just below the dome of the diaphragm. A single oral dose of $25-50 \mathrm{mg}$ atenolol (AstraZeneca Pharmaceuticals, London, UK) was administered $4 \mathrm{~h}$ before cardiac CT scanning if the pre-scan heart rate exceeded 80 beats/min. No additional medication was given if the heart rate did not decrease sufficiently following this dose.

CT examinations were contrast enhanced using our routine clinical contrast medium injection protocol. The delay time before acquiring data after the start of the injection of contrast medium was determined by test-bolus injection of $15-20 \mathrm{ml}$ of a non-ionic contrast medium (Iopamidol, $370 \mathrm{mg} / \mathrm{ml}$ Iopamiron, Bayer Yakuhin, Osaka, Japan) at $4 \mathrm{ml} / \mathrm{s}$ through a $20 \mathrm{G}$ intravenous antecubital catheter, followed by $20 \mathrm{ml}$ of saline, using a dual-syringe injector (Stellant, Medrad, Indianola, PA). The peak time of test bolus enhancement as measured by repetitive scanning at the level of the aortic root, was used as the delay time. After the test bolus, adenosine infusion was started at $140 \mu \mathrm{g} / \mathrm{kg} / \mathrm{min}$. A retrospectively gated scan was obtained $3 \mathrm{~min}$ after the initiation of the infusion [13]. Actual contrast enhancement was achieved by injecting $60-70 \mathrm{ml}$ of contrast medium, followed by $30 \mathrm{ml}$ of saline. All injections were delivered at $4 \mathrm{ml} / \mathrm{s}$. Throughout the infusion, patient symptoms, heart rate, blood pressure, and the electrocardiogram were monitored by a cardiologist. Immediately after the stress CT, the adenosine infusion was discontinued.

Adenosine stress scanning was performed in 10 patients; a rest scan was not performed for these patients. A rest scan was performed in eight patients in the same protocol; adenosine stress scan was not performed for these patients.

\section{Dual-energy post-processing}

From a single dual-energy CT data acquisition, three different image reconstructions were performed, with reconstructed section width of $0.75 \mathrm{~mm}$ and reconstruction increments of $0.4 \mathrm{~mm}$ using the routine dual-energy reconstruction algorithm implemented on the scanner platform. The first set of transverse gray-scale images aimed to optimize spatial and contrast resolution by merging $70 \%$ of the $140 \mathrm{kV}$ spectrum and $30 \%$ of the $100 \mathrm{kV}$ spectrum. These data sets were used for clinical coronary CTA interpretation of coronary artery morphology for stenosis detection and grading; the term ' $120 \mathrm{kV}$ composite image' used in the present study refers to the $120 \mathrm{kV}$ images. Another image set was based only on the low $\mathrm{kV}(100 \mathrm{kV}) \mathrm{X}$-ray spectrum, and yet another only on the $140-\mathrm{kV}$ X-ray spectrum. The iodine map was analyzed by determining the iodine content within the tissue, based on the unique X-ray absorption characteristics of this element at different $\mathrm{kV}$ levels [14, 15].

Coronary enhancement ratio (CER)

Coronary enhancement was determined in each patient in Hounsfield units (HU) as the mean contrast attenuation determined at regions of interest (ROIs) positioned on the aortic root, and at the right coronary artery (RCA), the left anterior descending artery (LAD), and the left circumflex artery (LCX). Each ROI was set as large as possible without including coronary wall, plaque, or calcifications, and was located at the same site on both the iodine map and the $120 \mathrm{kV}$ images. ROIs were not set at points with a diameter of $<3 \mathrm{~mm}$ or with significant stenosis. First, the coronary arteries were each divided into three portions (RCA: proximal, mid, and distal portions; LAD: left main trunk, proximal, and mid portions; LCX: left main trunk, proximal, and distal portions) on the basis of the AHA 15 segment model. Three ROIs in each portion were set at equal intervals. Next, the intra-coronary attenuation at nine different points was measured, and the mean values on both the iodine map and the $120 \mathrm{kV}$ images were calculated. Coronary enhancement ratio (CER) was used as an estimate of coronary enhancement, and was calculated by dividing the mean attenuation in the coronary artery on the iodine map or the $120 \mathrm{kV}$ images by the mean attenuation in the aortic root on the $120 \mathrm{kV}$ images.

\section{Analysis of coronary CT angiogram}

Coronary CT angiograms were analyzed using the $120 \mathrm{kV}$ data with a software (Virtual Place, AZE, Tokyo, Japan), based on a combination of transverse sections and automatically generated curved multiplanar reformatted images of the target vessels. A semi-automated vessel analysis tool was used for grading the severity of stenosis. The images were 
clinically interpreted by three experienced readers (two radiologists and one cardiologist) who reached consensus, using the American Heart Association 15 -segment model. Significant stenosis was defined as a reduction in diameter of more than $50 \%$.

Adenosine stress myocardial perfusion scintigraphy (MPS)

Stress/rest thallium-201 MPS was performed according to the American College of Cardiology (ACC)/ American Heart Association (AHA)/American Society of Nuclear Cardiology (ASNC) guidelines for the clinical use of cardiac radionuclide imaging [16]. For each patient, stress was induced pharmacologically through intravenous infusion of adenosine $(6 \mathrm{~min}$ infusion of $140 \mu \mathrm{g} \mathrm{kg}^{-1} \mathrm{~min}^{-1}$ ) as described by Nishimura et al. [17]. The patient's standard ECG, vital signs, and general conditions were continuously monitored during the stress protocol. Three minutes after the continuous infusion of adenosine, $111 \mathrm{MBq}$ thallium-201 was injected intravenously and flushed with saline. Early single photon emission tomography (SPECT) was performed $10 \mathrm{~min}$ after the adenosine stress test; late SPECT was performed $4 \mathrm{~h}$ after the early SPECT.

The SPECT images were acquired using a threeheaded SPECT system (GCA 9300; Toshiba, Tokyo, Japan). Tomographic reconstruction was performed using a standard filtered back-projection technique with a ramp filter to produce a transaxial tomogram. No scatter or attenuation correction was applied. From these transaxial tomograms, the long axis of the left ventricle was identified and oblique-angled tomograms were generated (i.e., vertical long-axis, shortaxis and horizontal long-axis tomograms).

The SPECT images were visually analyzed independently by one radiologist (M.N.) and one cardiologist (K.W.). The slices were displayed sequentially to assess the myocardial perfusion in each vascular territory [18]. The presence or absence of redistribution was visually judged in the $4 \mathrm{~h}$ images, which were used to determine whether ischemia was present or not.

Invasive coronary angiography (ICA)

ICA was performed by standard transfemoral arterial catheterization. A minimum of 8 projections were obtained (minimum of 5 views for the left coronary artery system and minimum of 3 views for the right coronary artery system). All ICA images were interpreted by two cardiologists (K.W., H.S.). Coronary artery segments were evaluated using a 15-segment AHA coronary tree model and were visually judged as having significant stenosis at 2 levels (i.e., if $>50 \%$ or $>75 \%$ luminal narrowing of the coronary artery diameter was present).

Statistical analysis

The Mann-Whitney $U$-test was used to examine differences in CER between stenotic and non-stenotic coronary arteries, between patients with and without stenotic coronary arteries, and between ischemia and non-ischemia on the MPS. The paired $t$-test was used to examine the differences in CER between the findings of the iodine map and those of $120 \mathrm{kV}$ images. Probability values $<0.05$ were considered significant.

\section{Results}

CER at adenosine stress

Coronary CTA under adenosine stress detected 12 stenotic lesions and one total occlusion in the coronary arteries in 6 of the 10 patients. The CERs for the one occluded artery and a diffuse calcified artery were not measured. We analyzed a total of 28 coronary arteries, including 12 stenotic and 16 nonstenotic coronary arteries. CER was evaluated on a per-coronary and per-patient basis.

On the iodine map, CER was significantly lower for stenotic coronary arteries $(0.77 \pm 0.06)$ than for nonstenotic coronary arteries $(1.07 \pm 0.33)(P<0.001)$. On the $120 \mathrm{kV}$ images, there was no difference in CER between stenotic $(0.87 \pm 0.09)$ and non-stenotic coronary arteries $(0.87 \pm 0.08)$ (Fig. 1$)$. In the case of stenotic coronary arteries, CER was significantly lower on the iodine map than on the $120 \mathrm{kV}$ images $(P<0.005)$. In the case of non-stenotic coronary arteries, CER was significantly higher on the iodine map than on the $120 \mathrm{kV}$ images $(P=0.02)$ (Fig. 2). CER of less than 0.85 separated stenotic from non-stenotic coronary arteries with a positive predictive value of $79 \%$ and a negative predictive value of $93 \%$. 


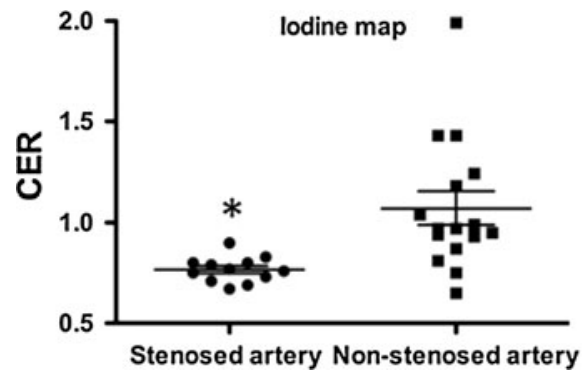

Fig. 1 Scatter plot shows the coronary enhancement ratio (CER) for stenotic and non-stenotic coronary arteries under adenosine stress. Horizontal long line represents the mean value and the upper and lower short lines the standard error of the mean. On the iodine map (left), CER was significantly less

Based on analyses of each patient, we divided them into those with and without stenotic coronary arteries. On the iodine map, CER was significantly lower for patients with stenotic coronary arteries $(0.77 \pm 0.08)$ than for those without them $(1.17 \pm 0.32) \quad(P<$ $0.0001)$. On the $120 \mathrm{kV}$ images, there was no difference in CER between patients with $(0.87 \pm 0.08)$ and without stenotic coronary arteries $(0.88 \pm 0.09)$ (Fig. 3). CER of less than 0.85 separated patients with stenotic coronary arteries from those without with a positive predictive value of $100 \%$ and a negative predictive value of $86 \%$.

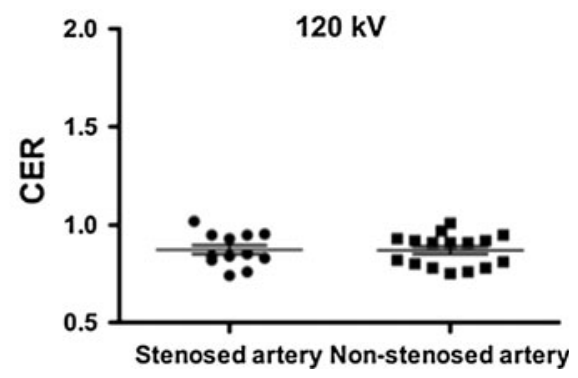

for stenotic coronary arteries than for non-stenotic coronary arteries $(* P<0.001)$. On the $120 \mathrm{kV}$ images (right), there was no difference in CER between stenotic and non-stenotic coronary arteries

Adenosine stress MPS was performed in 7 of 10 patients who had undergone adenosine stress CT. Adenosine stress MPS detected 9 ischemic territories out of 21 coronary territories in the 7 patients. CER on the iodine map was significantly lower for ischemic territories $(0.76 \pm 0.06)$ than non-ischemic territories $(0.95 \pm 0.21)(P=0.02)$. There was no difference in CER on the $120 \mathrm{kV}$ images between ischemic $(0.88 \pm 0.1)$ and non-ischemic territories $(0.85 \pm 0.08)$ (Fig. 4). When myocardial segments with a CER of less than 0.8 were diagnosed as ischemic territories, this criterion separated ischemic
Fig. 2 Comparison of the coronary enhancement ratio (CER) of stenotic and nonstenotic arteries on $120 \mathrm{kV}$ images and an iodine map under adenosine stress. In the case of stenotic coronary arteries (left), CER was significantly less on the iodine map than on the $120 \mathrm{kV}$ images (* $P<0.005)$. In the case of non-stenotic coronary arteries (right), CER was significantly greater on the iodine map than on the $120 \mathrm{kV}$ images (** $P=0.02$ )

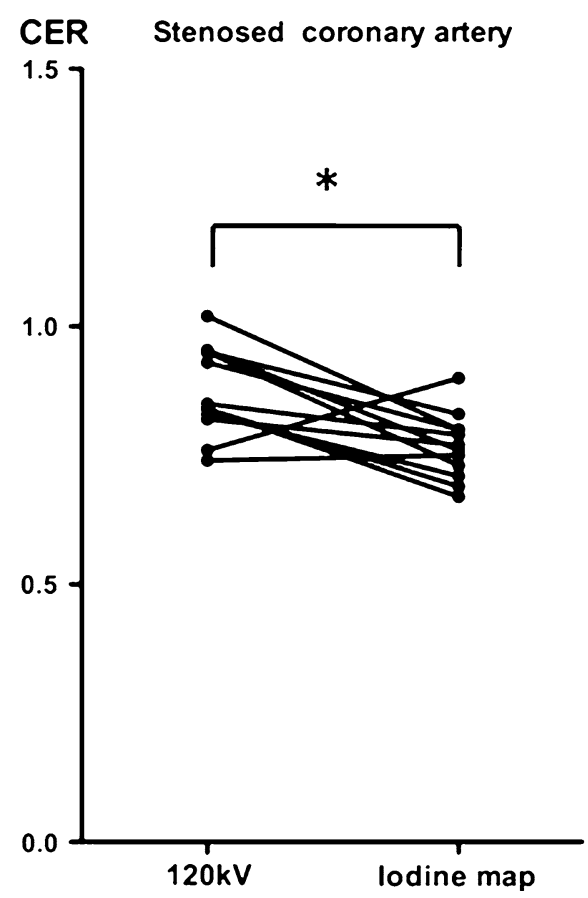

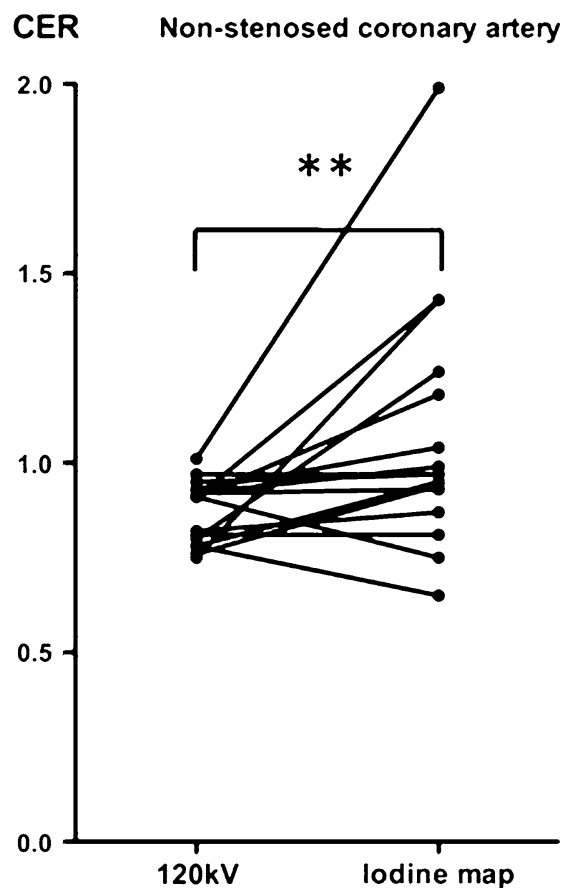




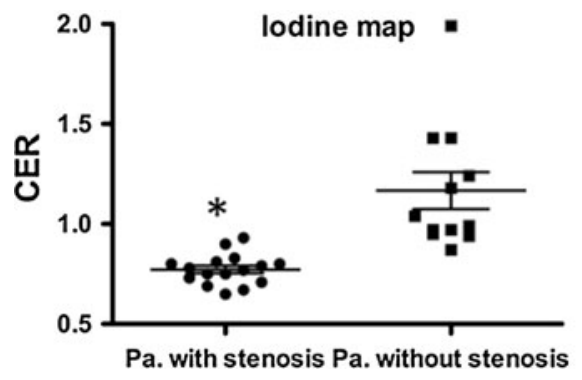

Fig. 3 Scatter plot shows the coronary enhancement ratio (CER) for patients with and without stenotic coronary arteries under adenosine stress. Horizontal lines are the same as in Fig. 1. On the iodine map (left), CER was significantly less for

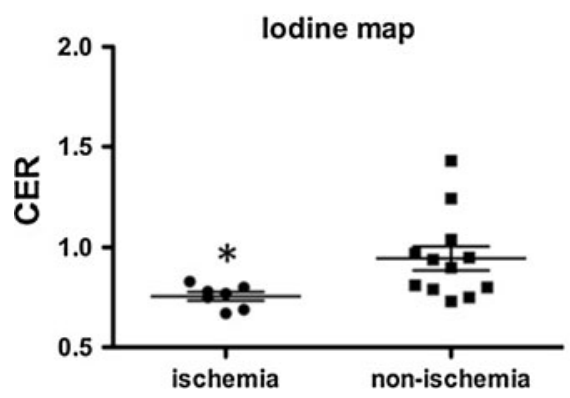

Fig. 4 Scatter plot shows the coronary enhancement ratio (CER) for ischemic territories and non-ischemic territories under adenosine stress. Horizontal lines are the same as in Fig. 1. On the iodine map (left), CER was significantly less for

from non-ischemic territories with the sensitivity of $86 \%$ and the specificity of $75 \%$.

ICA was performed in 5 of 10 patients who had undergone adenosine stress CT. ICA detected 5 moderately stenotic arteries (50-75\% stenosis) and 4 severely stenotic arteries ( $>75 \%$ stenosis) out of 15

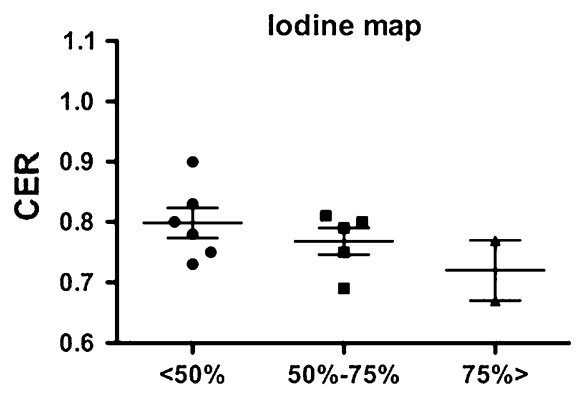

Fig. 5 Scatter plot shows the coronary enhancement ratio (CER) for territories with a coronary stenosis of $<50,50-75$, and $>75 \%$ on invasive coronary angiography under adenosine

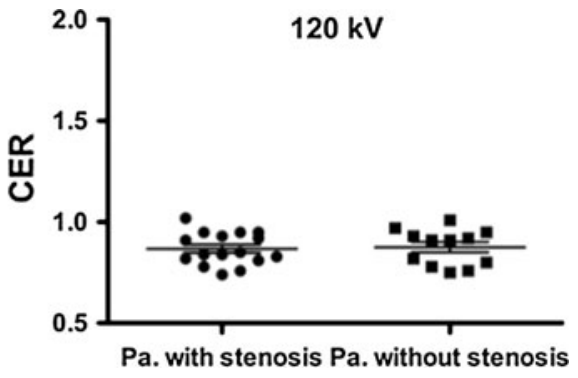

patients with stenotic coronary arteries than for those without them $(* P<0.0001)$. On the $120 \mathrm{kV}$ images (right), there was no difference in CER between patients with and without stenotic coronary arteries. $\mathrm{Pa}$ patients

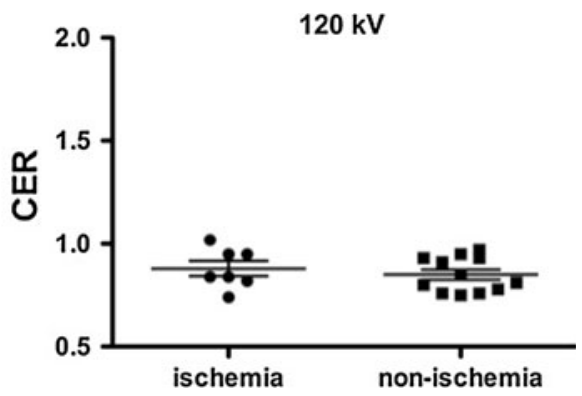

ischemic than for non-ischemic territories $(* P=0.02)$. On the $120 \mathrm{kV}$ images (right), there was no difference in CER between ischemic and non-ischemic territories

coronary arteries in 5 patients. On the iodine map, CER values for severely stenostic, moderately stenotic, and non-stenotic arteries were $0.72 \pm 0.07,0.77 \pm 0.05$, and $0.8 \pm 0.06$, respectively. On the $120 \mathrm{kV}$ images, the corresponding CER values were $0.83 \pm 0.01$, $0.85 \pm 0.1$, and $0.91 \pm 0.07$, respectively (Fig. 5).

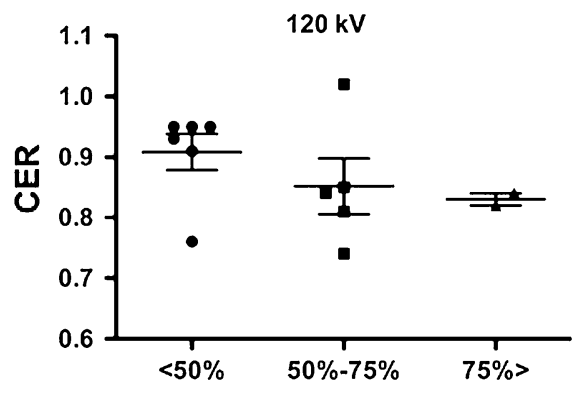

stress. On the iodine map (left), CER tended to decrease with progression of coronary stenosis. This tendency was not clearly observed at $120 \mathrm{kV}$ 
CER at rest

Coronary CTA at rest detected four stenotic lesions in the coronary arteries of 4 of the 8 patients. We analyzed a total of 24 coronary arteries, including 4 stenotic and 20 non-stenotic. On the iodine map, there was no difference in CER between stenotic $(0.81 \pm 0.1)$ and non-stenotic coronary arteries $(0.83 \pm 0.1)$. On the $120 \mathrm{kV}$ images, there was no difference in CER between stenotic $(0.95 \pm 0.04)$ and non-stenotic coronary arteries $(0.89 \pm 0.1)$. In the case of non-stenotic coronary arteries, there was no difference in CER findings between the iodine map and the $120 \mathrm{kV}$ images.

Coronary images on the iodine map and the $120 \mathrm{kV}$ images

Figures 6 and 7 show stenotic and non-stenotic coronary artery images on the iodine map and the $120 \mathrm{kV}$ images of representative cases.

\section{Discussion}

The dual-source CT scanner has opened new fields for dual-energy contrast-enhanced applications, since the two orthogonally mounted detectors and tube arrays operate simultaneously and can be set to different tube potentials, enabling dual-energy $\mathrm{CT}$ acquisitions with minimal patient motion registration artifacts [10]. One tube can be set at a peak voltage of $80 \mathrm{kV}$, while the other can be set at a peak voltage of $140 \mathrm{kV}$, and the resultant data sets can be reformatted using the dedicated software at a workstation. Maps of iodinated contrast material content can be extracted without the need for complex image registration, which is invariably required with traditional singlesource CT, and generally requires separate acquisition of unenhanced and contrast-enhanced CT images $[19,20]$. Dual-energy CT is used to map iodine distribution at a single time point; and the term iodine map is used in the present study. We hypothesized that the iodine map could detect alterations in coronary flow during adenosine stress and rest, since it emphasizes the degree of iodine distribution in the coronary arteries shortly after arrival of the contrast material bolus.

We propose the use of CER and our DE-CT analysis system as a simple and objective index for assessment of intra-coronary attenuation. During adenosine stress, CER on the iodine maps was significantly less for ischemic territories and stenotic arteries than for non-ischemic territories and nonstenotic arteries, whereas CER values for the two groups on the $120 \mathrm{kV}$ images were similar. Low CER value $(<0.85)$ on the iodine map can be used to diagnose stenotic coronary arteries with a high
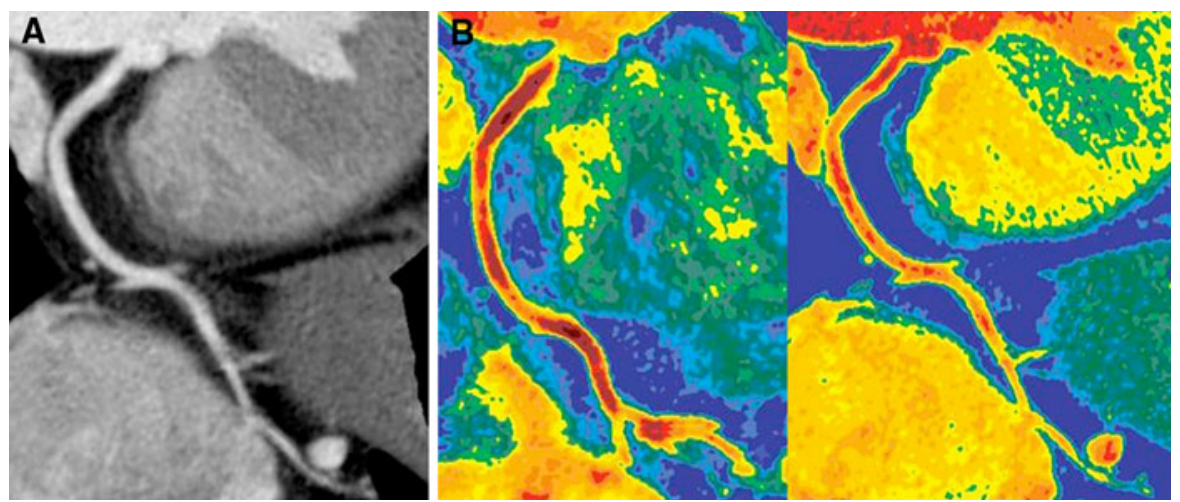

Fig. 6 Coronary images during adenosine stress in a 71-yearold female with normal coronary arteries. a Curved multiplanar reformatted image at $120 \mathrm{kV}$ shows no stenosis and no plaque in the right coronary artery. b Colored coronary images demonstrate higher attenuation within the coronary artery on the iodine map (right) than on the $120 \mathrm{kV}$ image (left). The average attenuation for the right coronary artery was $287 \mathrm{HU}$ on the $120 \mathrm{kV}$ images and $444 \mathrm{HU}$ on the iodine map. The CER value was 0.8 on the $120 \mathrm{kV}$ images and 1.24 on the iodine map. The color scale uses a gradient of warm colors, classified into six steps by Hounsfield units (HU) as follows: 150-200, 201-250, 251-300, 301-350, 351-400, 401-450 HU. Yellow indicates the lowest attenuation and dark red indicates the highest 


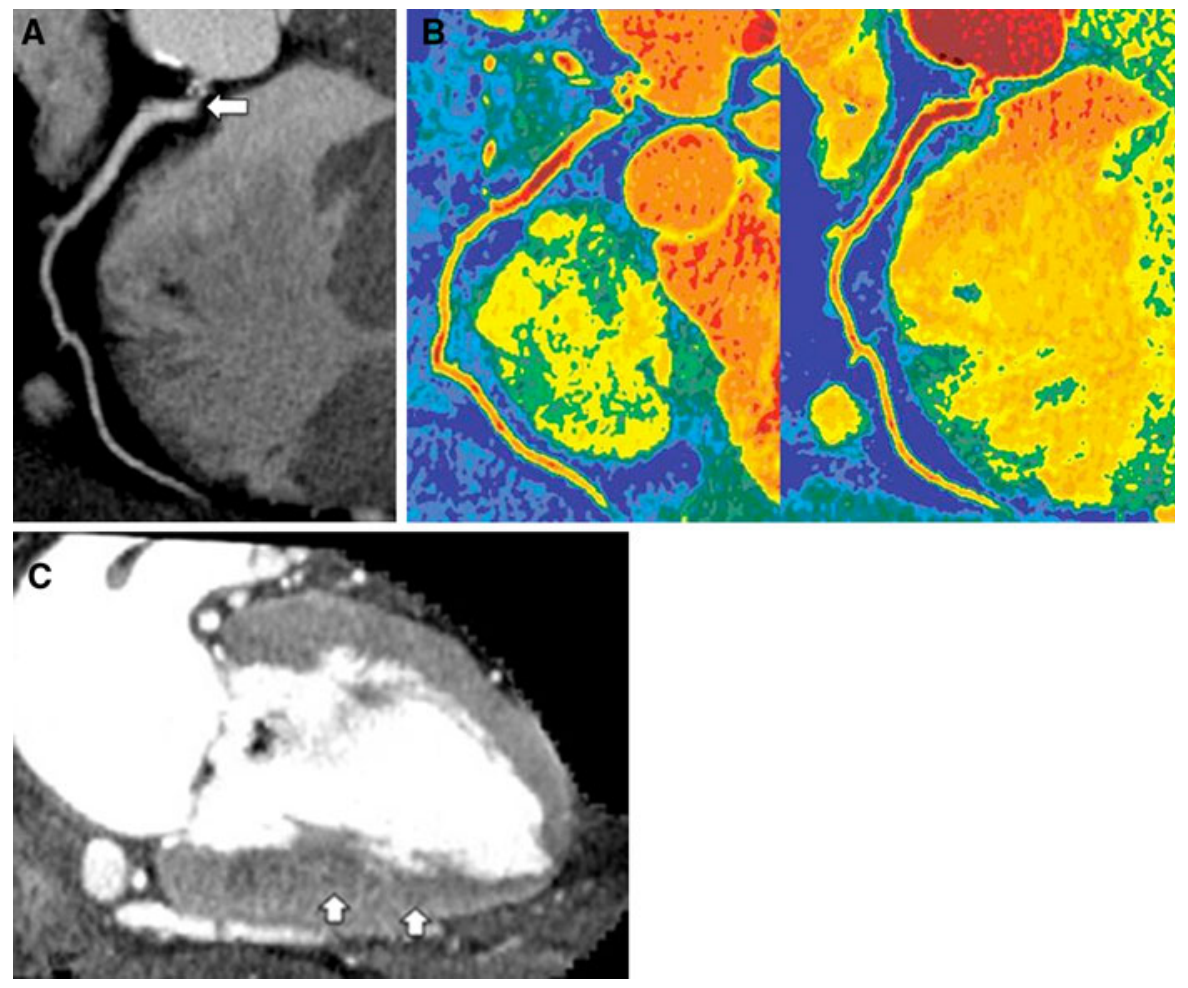

Fig. 7 Coronary and myocardial images during adenosine stress in a 55-year-old male with severe stenosis of the right coronary artery. a Curved multiplanar reformatted image at $120 \mathrm{kV}$ shows severe ostial stenosis of the right coronary artery (arrow). b Colored coronary images demonstrate higher attenuation within the coronary artery on the $120 \mathrm{kV}$ images (left) than on the iodine map (right). The average attenuation for the right coronary artery was $338 \mathrm{HU}$ on the $120 \mathrm{kV}$ images

positive predictive value and non-stenotic coronary arteries with a high negative predictive value. Furthermore, use of CER separated ischemic territories from non-ischemic territories with a high diagnostic accuracy, in comparison with the results of stress MPS. CER tended to progressively lower with a coronary stenosis of $<50 \%, 50-75 \%$, and $>75 \%$ on ICA. Lower CER $(<0.8)$ is strongly related to the physiological significance of $\mathrm{CAD}$, and is clinically important in determining treatment strategy in patients with CAD. Meijboom, et al. reported that anatomical assessment of coronary stenosis determined by CT coronary angiography does not correlate well with functional assessment of FFR [21]. However, our analytical technique is the first noninvasive means of overcoming the disadvantage of functional assessment of coronary artery flow using CT. and $318 \mathrm{HU}$ on the iodine map. The CER value was 0.82 on the $120 \mathrm{kV}$ images and 0.77 on the iodine map. The color scale used is the same as that in Fig. 4b. c Vertical long-axis slice (myocardial image) reconstructed from the same data as the $120 \mathrm{kV}$ coronary angiogram shows a region of subendocardial hypo-enhancement in the inferior wall (arrows), suggestive of myocardial ischemia

In addition, colored coronary images demonstrate high attenuation in non-stenotic coronary arteries and relatively low attenuation in stenotic coronary arteries. This agrees with the fact that adenosine stress induces hyperemia in normal coronary arteries and relative hypo-perfusion in significantly stenotic coronary arteries [22], as shown by fractional flow reserve (FFR) [23, 24] and coronary flow reserve [25, 26], which are well-established indices for determining the functional severity of coronary stenosis. Wilson et al. reported the changes in coronary blood flow velocity after progressively greater doses of intracoronary adenosine; the resulting larger doses of adenosine caused prolonged hyperemia [22]. We suspect that the change in coronary blood flow velocity may affect the degree of intracoronary enhancement on the iodine map. 
Blankstein et al. recently reported that adenosine stress CT identifies stress-induced myocardial perfusion defects with a diagnostic accuracy comparable to that of SPECT [13]. Under resting conditions, in the absence of critical CAD, myocardial perfusion is higher in the subendocardium than in the epicardium [27]. While this endocardial/epicardial flow ratio is maintained in myocardium supplied by normal epicardial arteries during the administration of adenosine, it is decreased in myocardium supplied by stenotic epicardial arteries. This results in relatively low attenuation in subendocardium and high attenuation in epicardium in ischemic myocardium, as reported in recent myocardial perfusion CT studies [28, 29]. As indicated in Fig. 5c, adenosine stress myocardial images on the iodine map could detect areas of subendocardial hypo-enhancement in four of six patients with stenotic coronary arteries. CER on the iodine map in these territories was significantly reduced (mean, 0.73). These findings suggest that lower CER reflects flow reduction in stenotic epicardial arteries under adenosine stress.

Our study has several significant limitations. First, the retrospective nature of our analysis in a relatively small, inhomogeneous clinical patient cohort limits systematic appraisal of our approach to routine, clinical detection of CAD based on DE-CT. In the present study, 10 patients who underwent adenosine stress DE-CT and 8 patients who underwent DE-CT at rest as a control group were enrolled. The adenosine stress group included six patients $(60 \%)$ with heart rate greater than $80 / \mathrm{min}$, while only one patient $(13 \%)$ in the rest CT group had a heart rate greater than $80 / \mathrm{min}$, i.e. the heart rate differed between the two groups. We recommend that the CER for non-stenotic arteries on the $120 \mathrm{kV}$ images be considered the baseline for intracoronary attenuation. Since this value was almost the same during adenosine-stress $(0.87 \pm 0.08)$ as at rest $(0.89 \pm 0.1)$, CER can be considered a reasonable objective index that is generally unaffected by heart rate variation. As a preliminary test for this study, we measured CER for 36 patients with normal coronary arteries on coronary CTA at rest using a single-source 64-slice MDCT. The CER value for a total of 108 normal coronary arteries of the 36 patients was $0.92 \pm 0.04$ (mean $\pm \mathrm{SD}$ ), and slightly higher than the mean CER (0.87) for non-stenotic coronary arteries during adenosine-stress on the $120 \mathrm{kV}$ images for the patients with CAD.
Second, because the dual-energy mode requires separate control of the two tubes, the temporal resolution of DE-CT cardiac acquisition is currently reduced to "only" $165 \mathrm{~ms}$, the same as for conventional single-source 64-slice CT. In addition, the heart rate tends to increase under adenosine stress. Coronary angiograms acquired under adenosine stress may be strongly affected by motion artifact because of limitations in temporal resolution and high heart ratio. Therefore, the iodine map cannot be used to assess the morphological severity of coronary stenosis. Steigner et al. recently reported that crosssectional contrast gradient in normal coronary artery was analyzed by prospectively ECG-gated single heart beat data obtained from 320-MDCT [30]. The assessment of cross-sectional contrast gradient may be related to coronary flow, and requires a high temporal resolution (single heart beat) and a high spatial resolution. On the other hand, scan time using retrospective ECG-gating in the present study was more than 7 or 8 heartbeats. Coronary enhancement on the iodine map may be affected by the addition of numerous cardiac cycles. Accordingly, CER on the iodine map may be less related to the cross-sectional contrast gradient in coronary arteries.

Dual-source CT was performed with parameters of 140 and $80 \mathrm{kV}$, which are often used because DE-CT requires a large difference in the tube voltage energy. However, iodine maps with parameters of 140 and $80 \mathrm{kV}$ have low signal-to-noise ratios, as a result of which diagnostic accuracy decreases, particularly for myocardial ischemia. Use of 140 and $100 \mathrm{kV}$ can double the dose (mAs), compared with the use of 140 and $80 \mathrm{kV}$. Consequently, use of 140 and $100 \mathrm{kV}$ decreases imaging noise and increases the reliability of iodine maps. Schenzle et al. recently reported that there was no difference in the effective radiation dose measured with the thermoluminescent detectors between dual-energy mode at 100 and $140 \mathrm{kV}$ and the standard $120 \mathrm{kV}$ scans, and that dual-energy CT is feasible without additional dose [31].

\section{Conclusion}

To the best of our knowledge, ours is the first noninvasive analytical technique for assessment of coronary artery flow using cardiac CT. CER on the iodine map is a candidate method for demonstration 
of alteration in coronary artery flow under adenosine stress, which is related to the physiological significance of coronary artery disease.

Open Access This article is distributed under the terms of the Creative Commons Attribution Noncommercial License which permits any noncommercial use, distribution, and reproduction in any medium, provided the original author(s) and source are credited.

\section{References}

1. Leschka S, Alkadhi H, Plass A et al (2005) Accuracy of MSCT coronary angiography with 64-slice technology: first experience. Eur Heart J 26:1482-1487

2. Raff GL, Gallagher MJ, Oneill WW, Goldstein JA (2005) Diagnostic accuracy of noninvasive coronary angiography using 64-slice spiral computed tomography. J Am Coll Cardiol 46:552-557

3. White CW, Wright CB, Doty DB et al (1984) Does visual interpretation of the coronary arteriogram predict the physiologic importance of a coronary stenosis? N Engl J Med 310:819-824

4. Kern MJ, Lerman A, Bech JW et al (2006) Physiological assessment of coronary artery disease in the cardiac catheterization laboratory: a scientific statement from the American Heart Association Committee on Diagnostic and Interventional Cardiac Catheterization, Council on Clinical Cardiology. Circulation 114:1321-1341

5. Tobis J, Azarbal B, Slavin L (2007) Assessment of intermediate severity coronary lesions in the catheterization laboratory. J Am Coll Cardiol 49:839-848

6. Silber S, Albertsson P, Aviles FF et al (2005) Guidelines for percutaneous coronary interventions. The task force for percutaneous coronary interventions of the European society of cardiology. Eur Heart J 26:804-847

7. Smith SC Jr, Feldman TE, Hirshfeld JW Jr et al (2006) ACC/AHA/SCAI 2005 guideline update for percutaneous coronary intervention: a report of the American College of Cardiology/American Heart Association Task Force on Practice Guidelines (ACC/AHA/SCAI Writing Committee to Update 2001 Guidelines for Percutaneous Coronary Intervention). Circulation 113:e166-e286

8. Weustink AC, Meijboom WB, Mollet NR et al (2007) Reliable high-speed coronary computed tomography in symptomatic patients. J Am Coll Cardiol 50:786-794

9. Brodoefel H, Burgstahler C, Tsiflikas I et al (2008) Dualsource CT: effects of heart rate, heart rate variability, and calcification on image quality and diagnostic accuracy. Radiology 247:346-355

10. Johnson TR, Krauss B, Sedlmair M et al (2007) Material differentiation by dual energy CT: initial experience. Eur Radiol 17:1510-1517

11. Zhang LJ, Zhao YE, Wu SY et al (2009) Pulmonary embolism detection with dual-energy CT: experimental study of dual-source CT in rabbits. Radiology 252:61-70

12. Ruzsics B, Lee H, Zwerner PL et al (2008) Dual-energy $\mathrm{CT}$ of the heart for diagnosing coronary artery stenosis and myocardial ischemia-initial experience. Eur Radiol 18: 2414-2424

13. Blankstein R, Shturman LD, Rogers IS et al (2009) Adenosine-induced stress myocardial perfusion imaging using dual-source cardiac computed tomography. J Am Coll Cardiol 54:1072-1084

14. Flohr TG, McCollough CH, Bruder H et al (2006) First performance evaluation of a dual-source CT (DSCT) system. Eur Radiol 16:256-268

15. Riederer SJ, Mistretta CA (1977) Selective iodine imaging using K-edge energies in computerized x-ray tomography. Med Phys 4:474-481

16. Klocker FJ, Baird MG, Lorell BH et al (2003) ACC/AHA/ ASNC guidelines for the clinical use of cardiac radionuclide imaging. Circulation 108:1404-1418

17. Nishimura S, Mahmarian JJ, Boyce TM, Verani MS (1991) Quantitative thallium-201 single-photon emission computed tomography during maximal pharmacologic coronary vasodilatation with adenosine for assessing coronary artery disease. J Am Coll Cardiol 18:736-745

18. Cerqueira MD, Weissman NJ, Dilsizian V et al (2002) Standardized myocardial segmentation and nomenclature for tomographic imaging of the heart: a statement for healthcare professionals from the Cardiac Imaging Committee of the Council on Clinical Cardiology of the American Heart Association. J Nucl Cardiol 9:240-245

19. Chung MJ, Gao JM, Im JG, Lee KS, Kim KG, Park JH (2004) CT perfusion image of the lung: value in detection of pulmonary embolism in a porcine model. Invest Radiol 39:633-640

20. Wildberger JE, Klotz E, Ditt H, Spuntrup E, Mahanken AH, Gunther RW (2005) Multislice computed tomography perfusion imaging for visualization of acute pulmonary embolism: animal experience. Eur Radiol 15:1378-1386

21. Meijboom WB, Van Mieghem CAG, Van Pelt $\mathrm{N}$ et al (2008) Comprehensive assessment of coronary artery stenoses: computed tomography coronary angiography versus conventional coronary angiography and correlation with fractional flow reserve in patients with stable angina. J Am Coll Cardiol 52:636-643

22. Wilson RF, Wyche R, Christensen B, Zimmer S, Laxon D (1990) Effect of adenosine on human coronary arterial circulation. Circulation 82:1595-1606

23. Pijils NH, De Bruyne B, Peels K et al (1996) Measurement of fractional flow reserve to assess the functional severity of coronary-artery stenoses. N Engl J Med 334:17031708

24. Bishop AH, Samady H (2004) Fractional flow reserve: critical review of an important physiologic adjunct to angiography. Am Heart J 147:792-802

25. Baumgart D, Haude M, Liu F, Ge J, Goerge G, Erbel R (1998) Current concepts of coronary flow reserve for clinical decision making during cardiac catheterization. Am Heart J 136:136-149

26. Kern MJ (2000) Coronary physiology revisited: practical insights from the cardiac catheterization laboratory. Circulation 101:1344-1351

27. Stoll M, Quentin M, Molojavyi A, Thamer V, Decking UK (2008) Spatial heterogeneity of myocardial perfusion predicts local potassium channel expression and action potential duration. Cardiovasc Res 77:489-496 
28. Kurata A, Mochizuki T, Koyama Y et al (2005) Myocardial perfusion imaging using adenosine triphosphate stress multislice spiral computed tomography: alternative to stress myocardial perfusion scintigraphy. Circ J 69:550-557

29. Nagao M, Matsuoka H, Kawakami H et al (2008) Quantification of myocardial perfusion by contrast-enhanced 64-slice MDCT: characterization of ischemic myocardium. AJR 191:19-25
30. Steigner ML, Mitsouras D, Whitmore AG et al (2010) Iodinated contrast opacification gradients in normal coronary arteries imaged with prospectively ECG-gated single heart beat 320-detector row computed tomography. Circ Cardiovasc Imaging 3:179-186

31. Schenzle JC, Sommer WH, Neumaier K et al (2010) Dual energy CT of the chest: how about the dose? Invest Radiol 45:347-353 